\title{
The structure of brushes made of dendrimers: Recent advances
}

\author{
Cheng-Wu Li, ${ }^{1}$ Holger Merlitz,,${ }^{1,2, *}$ Chen-Xu Wu,${ }^{1, \dagger}$ and Jens-Uwe Sommer ${ }^{2}$ \\ ${ }^{1}$ Research Institute for Biomimetics and Soft Matter, Fujian Provincial Key Laboratory for Soft Functional Materials Research, \\ Department of Physics, Xiamen University, Xiamen 361005, China \\ ${ }^{2}$ Leibniz-Institut für Polymerforschung Dresden, 01069 Dresden, Germany
}

(Dated: March 3, 2016)

\begin{abstract}
In recent publications, a couple of theoretical models, ranging from simple scaling approaches and box-like mean-field models to self-consistent field techniques, have been proposed to describe the structure of brushes that are composed of dendrimers. In this work, we carry out molecular dynamics simulations in various density regimes, and compare the resulting data with predictions of the latest theoretical models. This study enables us to access the ranges of validity and accuracy of the respective models, as well as their shortcomings. Significant differences in the predicted conformations of the dendrimers are observed and analyzed in the context of their elastic properties in the different models. This work offers guidelines for future research directions, which may lead to further progress regarding the understanding of branched polymer brushes.
\end{abstract}

\section{INTRODUCTION}

Brushes made of linear chains have been studied thoroughly during the last decades, and their physical properties and structures are fairly well understood. To the contrary, the detailed structures of brushes made of branched polymers is still a matter of intense research. In the present work, we highlight recent progress in the modeling and simulation of brushes made of grafted dendrimers. These dendrimer brushes are interesting for a couple of reasons: Since they offer an enhanced number of end groups which could be functionalized, they are regarded promising candidates for environmentresponsive surface layers [1-6]. They are expected to offer improved anti-inflammatory or anti-fouling properties, i.e. barriers against a penetration by bacteria or proteins [7-9]. If grafted onto colloids, they lead to an improved stability against coagulation, featuring a thinner polymeric layer than colloids decorated with linear chains [10]. Last not least, branched polymer brushes are known to play vital roles in biological materials such as airway epithelia [11], are serving as anti-viral barriers [12] or potential high-efficiency drugdelivery systems [13].

Experimentally, the preparation of polymer brushes follows two approaches, denoted as grafting-from and graftingto techniques [14]. Dendrimers are synthesized via a stepcontrolled technique, and a large number of different dendritic polymers of low degree of polydispersity are now being made in the laboratories [15-18].

The present work will focus on advances of the theoretical understanding, the modeling and the simulation of dendrimer brushes. Comparisons of simple, Flory-Huggins based mean field models, both in continuum and on the lattice, with self-consistent field (SCF) techniques and molecular dynamics (MD) simulations will offer insights into basic conformational features and properties of these systems. We will focus on brushes, grafted onto planar substrate, and in good solvent, in which the molecules are highly swollen. In Sec. II,

\footnotetext{
*merlitz@posteo.de

$\dagger$ cxwu@xmu.edu.cn
}

we will first pay attention to brushes at low grafting densities, for which particularly simple and hence fully analytical formalisms have been derived. In Sec. III, these models are compared to MD simulation data, and their scaling behavior is evaluated. In Sec. IV, a couple of refined models are presented, which are assuming a dual layer structure of starlike brushes at higher densities and which account for the finite extensibility of the spacers. We will analyze systematic differences between these approaches in Sec. V, and particularly in Sec. V.2, in which conformational features of the dendrimers inside the brushes are analyzed. In Sec. VI, we shall critically discuss the progress achieved so far, and then speculate about possible directions which may lead toward future improvements.

\section{FULLY ANALYTICAL BRUSH MODELS}

For sparse brushes, in which the monomer densities and the stretching ratios of the spacers remain low, fully analytical models are available. Among these models we may distinguish between single phase box models, in which the monomer density profiles are uniform step-like functions, and SCF models, which usually assume parabolic SCF potentials. Fully analytical solutions for the brush heights and densities seem possible as long as the spacers (i.e. stems or branches of the grafted dendrimers) are inside the weak stretching regime, in which their elastic forces are well approximated by a linear Hooke-law. In the examples considered here, volumetric and entropic mixing effects are approximated by the leading order term of the virial expansion.

\section{II.1. Single phase box models}

Simple box models with uniform monomer densities, used to derive scaling laws of the brush height and concentration of dendrimer brushes, are generalizations of the classical Alexander-deGennes model $[19,20]$ and available since quite some time [21]. As has been pointed out by Kröger et al. [22], the validity of the resulting scaling laws is more often than not 
limited to narrow parameter windows and should therefore be routinely checked with computer simulations. However, recent modifications of boxlike models and SCF theories allow for higher accuracies and hence a superior understanding of the limitations of traditional scaling approaches.

According to the standard mean-field concept of neutral (uncharged) brushes, the total free energy, as a function of the brush height $H$, is composed of two contributions,

$$
F(H)=F_{\text {int }}(H)+F_{\text {el }}(H) .
$$

The interaction free energy may be taken from a FloryHuggins type mean-field model [23], or, more generally, approximated as a virial expansion in powers of the number density $c$,

$$
\frac{F_{\text {int }}}{V}=\frac{1}{2}\left(\mathrm{v} c^{2}+\mathrm{w} c^{3}+\cdots\right),
$$

with the system volume $V$, the excluded volume coefficient $\mathrm{v}$ and the three-body interaction coefficient $\mathrm{w}$. This expansion includes the free energy of mixing of the monomers with the solvent. Within the framework of the Flory-Huggins model, only the coefficient $\mathrm{v}$ accounts for enthalpic interactions between the monomers, and thus it depends on the temperature. In good solvent and at low densities, the leading order excluded volume term may suffice, while in $\Theta$-solvent the third order term would define the leading contribution. Here and in all subsequent equations, we express free energies in units of $k_{B} T$.

In a box model with uniform density, the number density $c$ and the brush height $H$ are related through

$$
c=\frac{M N}{V}=\frac{M N}{A H}=\frac{\sigma N}{H},
$$

with the number of monomers $N$ per grafted molecule, the total number $M$ of grafted molecules, the brush area $A$ and the grafting density $\sigma=M / A$, so that in good solvent

$$
F_{\text {int }}(H) \approx \mathrm{v} \frac{M N^{2} \sigma}{2 H} .
$$

Under weak stretching conditions of the spacers, the elastic free energy per molecule may be expressed with the help of a simple harmonic potential

$$
\frac{F_{\mathrm{el}}}{M}=\frac{1}{2} K H^{2}
$$

with the molecular spring-constant $K$ (for linear chains, $K=$ $3 / N$; note that we assume a unit bond length or monomer diameter here and in the following equations, unless stated otherwise). The free energy minimum is then obtained from

$$
\frac{\partial F}{\partial H} \stackrel{!}{=} 0=K H-\frac{\mathrm{v} \sigma N^{2}}{2 H^{2}}
$$

yielding

$$
H=\left(\frac{\mathrm{v}}{2}\right)^{1 / 3}\left(\frac{N^{2}}{K}\right)^{1 / 3} \sigma^{1 / 3}
$$

The first factor contains the excluded volume coefficient. Since it originates in a Flory theory, being valid on a lattice, it may, during comparisons with other theoretical models in continuum space or with molecular simulations, be used as a free fit parameter. The second factor contains the effective elasticity of the molecule and depends both on its architecture and the mode of stretching, i.e. the conformations of the molecules inside the brush. The third factor contains the scaling of the brush height as a function of the grafting density. More generally, using scaling concepts, the relation $H \sim \sigma^{(1-v) / 2 v}$ has been derived for linear brushes [19, 20], with the Flory exponent $v \approx 0.588 \approx 3 / 5$. For the monomer concentration inside the box we immediately get

$$
c=\left(\frac{2}{\mathrm{v}}\right)^{1 / 3}(N K)^{1 / 3} \sigma^{2 / 3} .
$$

Note that Eqs. 7 and 8 are fully general and valid for (uncharged) linear brushes as well as dendrimer brushes. Only the elasticity constant $K$ varies, depending on the dendrimer's architecture and also on the assumed stretching scenario, which will be discussed in the following section.

\section{II.2. Stretching scenarios}

While the equations derived in the previous section contain the yet unspecified spring constant $K$, the latter depends on the typical conformations of the molecules and is now to be evaluated for dendrimers in different stretching scenarios.

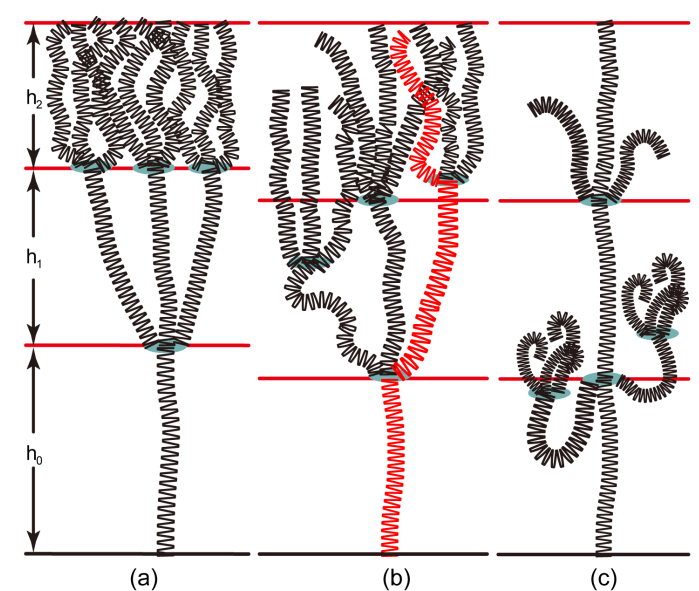

FIG. 1. Stretching scenarios: Schematic illustration of dendrimers composed of generation $g=2$ and functionality $q=3$. Each spacer (connection between branching points) consists of $N_{S}$ monomers, a thread (shown in red) is a path connecting the grafting point with one of the ends, thus containing $(g+1) N_{s}$ monomers. Considered are stretching scenarios: (a): The 'all up' scenario, in which the branching points of each generation occupy identical vertical positions; (b): The uniform stretching scenario, in which each elastic thread carries (on average) the same elastic energy; (c): The longest path stretching scenario, in which only a single thread is stretched up, while the remaining spacers are relaxed. 
Figure 1 (a) displays what we shall call the 'all up' conformation of a dendrimer: In this case, all free branches of the dendrimer are stretched up to the same height. Elementary algebra then yields the effective spring constant as

$$
K_{\mathrm{au}}=\frac{3 q^{g}}{N},
$$

which, with Eq. 7 and the total number of monomers per molecule,

$$
N=N_{S} \frac{q^{g+1}-1}{q-1},
$$

leads to

$$
H_{\mathrm{au}}=\left(\frac{\mathrm{v} \sigma}{6 q^{g}}\right)^{1 / 3} N=\left(\frac{\mathrm{v}}{6}\right)^{1 / 3} \sigma^{1 / 3} N_{S} \frac{q^{g+1}-1}{q-1} q^{-g / 3} .
$$

$N_{S}$ denotes the number of monomers in each spacer (note that in the present work we focus on symmetric dendrimers, in which $N_{S}$ is identical for each spacer). The corresponding concentration according to Eq. 8 is obtained as

$$
c_{\mathrm{au}}=\left(\frac{6}{\mathrm{v}}\right)^{1 / 3} \sigma^{2 / 3} q^{g / 3} .
$$

The 'all up' scenario is inconsistent in the sense that it would, if applied to all dendrimers of the brush, lead to a non-uniform monomer density profile, and the highest density would be observed in the upper section of the brush. Later we will discuss how this inconsistency is avoided within the mean-field model through the introduction of several dendrimer populations, some of which consisting of retracted molecules which occupy and fill up the lower sections of the brush. For the time being, we may simply assume that only a certain fraction of molecules contributes to the elastic free energy.

In another scenario, just a single thread of the dendrimer is stretched up to the brush height $H$, while all other strands are assumed to remain relaxed. This 'longest path' scenario, shown in Fig. 1 (c), has been discussed before [10, 24, 25], and it leads to the effective spring constant

$$
K_{\mathrm{lp}}=\frac{3}{(g+1) N_{S}},
$$

yielding

$$
H_{\mathrm{lp}}=\left(\frac{\mathrm{v}}{6}\right)^{1 / 3} \sigma^{1 / 3} N_{S}\left(\frac{q^{g+1}-1}{(q-1)}\right)^{2 / 3}(g+1)^{1 / 3}
$$

and

$$
c_{\mathrm{lp}}=\left(\frac{6}{\mathrm{v}}\right)^{1 / 3} \sigma^{2 / 3}\left(\frac{q^{g+1}-1}{(q-1)(g+1)}\right)^{1 / 3} .
$$

A third scenario has been applied before to model the conformations of free (non-grafted) dendrimers in solution [26, 27], and dendrimer conformations in brushes [22, 24]. For this 'uniform' stretching scenario, shown in Fig. 1 (b), the dendrimer is assumed to consist of $N /\left[(g+1) N_{S}\right]$ elastic units (or threads, one of them shown schematically in red), while every thread is assumed to carry (on average) the same elastic energy. This results in an effective elastic constant of

$$
K_{\text {uni }}=\frac{3 N}{(g+1)^{2} N_{S}^{2}},
$$

yielding

$$
H_{\text {uni }}=\left(\frac{\mathrm{v}}{6}\right)^{1 / 3} \sigma^{1 / 3} N_{S}\left(\frac{q^{g+1}-1}{(q-1)}\right)^{1 / 3}(g+1)^{2 / 3}
$$

and

$$
c_{\text {uni }}=\left(\frac{6}{\mathrm{v}}\right)^{1 / 3} \sigma^{2 / 3}\left(\frac{q^{g+1}-1}{(q-1)(g+1)}\right)^{2 / 3} .
$$

However, it is questionable whether or not the uniform stretching model, when applied to grafted dendrimers, can be made consistent with the force balance condition at the branching points.

\section{II.3. Analytical Gaussian SCF theory}

A first analytical SCF model of branched polymer brushes has been presented by Pickett [28] and subsequently been generalized by Polotsky et al. [29] and Zhulina et al. [30-32]. Assuming a low stretching and thus Gaussian elasticity of the molecules, this approach arrives at a parabolic self-consistent potential of the form

$$
U(z)=\frac{3}{2} \alpha^{2}\left(H^{2}-z^{2}\right),
$$

in which $z$ denotes the vertical brush coordinate, while the factor $\alpha$ depends on the architecture of the dendrimer. With symmetric stars this model yields [30]

$$
\alpha(g=1)=\frac{1}{N_{S}} \arctan \frac{1}{\sqrt{q}},
$$

or, for symmetric dendrimers of second generation [32],

$$
\alpha(g=2)=\frac{1}{N_{S}} \arctan \frac{1}{\sqrt{q(q+2)}} .
$$

The monomer density profiles turn out to be parabolic, too, with

$$
\phi_{\text {gauss }}(z)=\frac{3 \alpha^{2}}{4 v}\left(H^{2}-z^{2}\right),
$$

and the resulting brush heights are derived as

$$
H_{\mathrm{SCF}}=\left(\frac{2 \mathrm{v} \sigma N}{\alpha^{2}}\right)^{1 / 3}
$$

thus exhibiting the same general scaling behavior $H \sim N \sigma^{1 / 3}$ as the box models of the previous section. This model has also 
been generalized to dendrimers, in which the spacer lengths differ with the generation of the dendrimer - a complication we are not going to discuss further. Because the analytical SCF-theory relies on the Gaussian elasticity of the spacers, it is restricted to the regimes of low and moderate grafting densities.

\section{II.4. SCF theory with self-avoiding random walks}

Recently, Romeis et al. have suggested another SCF theory of brushes in good solvent [33]. Based on the ordinary strong stretching approximation of (linear) polymer chains, they replaced the Gaussian elasticity with an elasticity that results from the self-avoiding random walk, achieving a more realistic representation of the conformational properties in good solvent. In this way, they derived a density profile of the functional form

$$
\phi_{\mathrm{SARW}}(z)=\phi_{o}\left(1-\left(\frac{z}{H}\right)^{1 /(1-v)}\right)^{3 v-1}
$$

with $v \approx 0.588$ in good solvent and $v=1 / 2$ in $\Theta$-solvent. We note that the brush height cannot be expressed in terms of a closed analytical expression, but its scaling properties, $H \sim N \sigma^{(1-v) / 2 v}$, are shown to be rigorously satisfied. Once the unknown numerical pre-factor is determined through a fit to the height of a particular brush, a systematic generalization to varying grafting densities is thus trivial. The concentration $\phi(z=0)=\phi_{o}$ then follows from normalization conditions. The formalism is based on the analysis of brushes made of linear polymers, and a generalization to dendrimer brushes is pending. In this context it may be interesting to note that within $\Theta$-solvent, when the scaling exponent is $v=1 / 2$, this formalism yields identical density profiles as the previously described analytical SCF model. A generalization of the latter to branched brushes in good solvent, combined with the selfavoiding random walk model, may thus be feasible. Since none of these models account for a finite extensibility of the spacers, they are generally restricted to brushes with fairly low monomer densities.

\section{COMPARISON WITH MD-SIMULATIONS}

In our MD simulations, the polymers were coarse-grained, freely jointed bead-springs, as first introduced by Kremer and Grest [34]. Details of the simulation procedure have been presented before $[35,36]$, so that a brief summary of the interaction model has to suffice here:

The beads are spherical monomers which interact via a shifted Lennard-Jones (LJ) potential

$$
U_{\mathrm{LJ}}(r)=4 \varepsilon\left[\left(\frac{d}{r}\right)^{12}-\left(\frac{d}{r}\right)^{6}-\left(\frac{d}{r_{c}}\right)^{12}+\left(\frac{d}{r_{c}}\right)^{6}\right]
$$

where $d=1$ stands for the bead diameter and $\varepsilon=1$ defines the strength of the interaction. The parameter $r_{c}$ is the cutoff distance. It is easily verified that without any cutoff $\left(r_{c} \rightarrow \infty\right)$ this potential has a minimum at $r_{\min }=2^{1 / 6} d$ with the depth $U_{\mathrm{LJ}}\left(r_{\min }\right)=-\varepsilon$. In turn, once a cutoff $r_{c}=2^{1 / 6} d$ is implemented, the attractive contribution to this potential is eliminated and in this way monomers in an implicit, athermal solvent are simulated. This repulsive LJ-potential is also referred to as Weeks-Chandler-Anderson (WCA) potential [37] and was applied within all simulations of this work. The connectivity between monomers was enforced by a finite extensible nonlinear elastic (fene) potential [34], defined as

$$
U_{\mathrm{FENE}}(r)=-0.5 K R_{0}^{2} \ln \left[1-\left(r / R_{0}\right)^{2}\right],
$$

with a stiff spring constant $K$ and a maximum allowed bond length of $R_{0}=1.5 \mathrm{~d}$. The range of bond lengths has never been exhausted, once the brushes had reached their equilibrium conformations: Bond lengths then varied between $a \approx 0.97$ (relaxed spacers) and $a \approx 1.01$ (in the case of highly elongated stems at high grafting densities). The simulations were carried out with the open source LAMMPS molecular dynamics package [38].

To test the different brush models, starlike polymer brushes of different functionalities $q$ were first simulated at low grafting density $\sigma=0.025$, at which the elastic tension of the spacers remains moderate. Figure 2 displays vertical density profiles, i.e. number densities $c$ as a function of the vertical coordinates $Z$, both after a corresponding scaling. The MD simulations (data points) are scaling best if the axes are scaled according to the the 'all up' model, i.e. Eq. 11 and 12, respectively (upper panel; note that $N_{S}$ and $g$ were constant in this set of systems and hence not shown explicitly in the scaling terms). The profiles are fairly accurately falling onto a single master curve, which is less so with the profiles evaluated from the analytical SCF theory. We note that we have used the excluded volume $\mathrm{v}$ as a free parameter to fit the SCF height $H$ to the inflection point of the profile of the $q=2$-brush (dashed vertical line). The fit was achieved with the choice $\mathrm{v}=0.8$. For a discussion of different approaches to the definition of brush heights, we refer to the Sec. V.1.

The SCF profiles display best scaling in the longest path stretching scenario, in which the MD data do not fall onto a master curve. It is interesting to note that a scaling according to the longest path model has been reported to hold for brushes which were simulated with the numerical Scheutjens-Fleer (SF)-SCF method [39] at low grafting densities [24]. This particular scaling behavior is therefore not exclusively a result of the approximations used in the analytical SCF method.

When applying the uniform stretching model to the density profiles, neither the MD simulations nor the SCF data are scaling well.

Since the MD simulations are generating the coordinates of all individual molecules, it is worth taking a closer look at their conformations. The average stretching ratios of the grafted spacers (stems), defined as the ratios $r=h / N_{S}$ of the vertical heights of the stems and their contour length, are increasing monotonously from $r(q=2)=0.34$ to $r(q=$ $5)=0.43$, and the stretching ratios of the most extended free branch per molecule vary between $r(q=2)=0.24$ and 

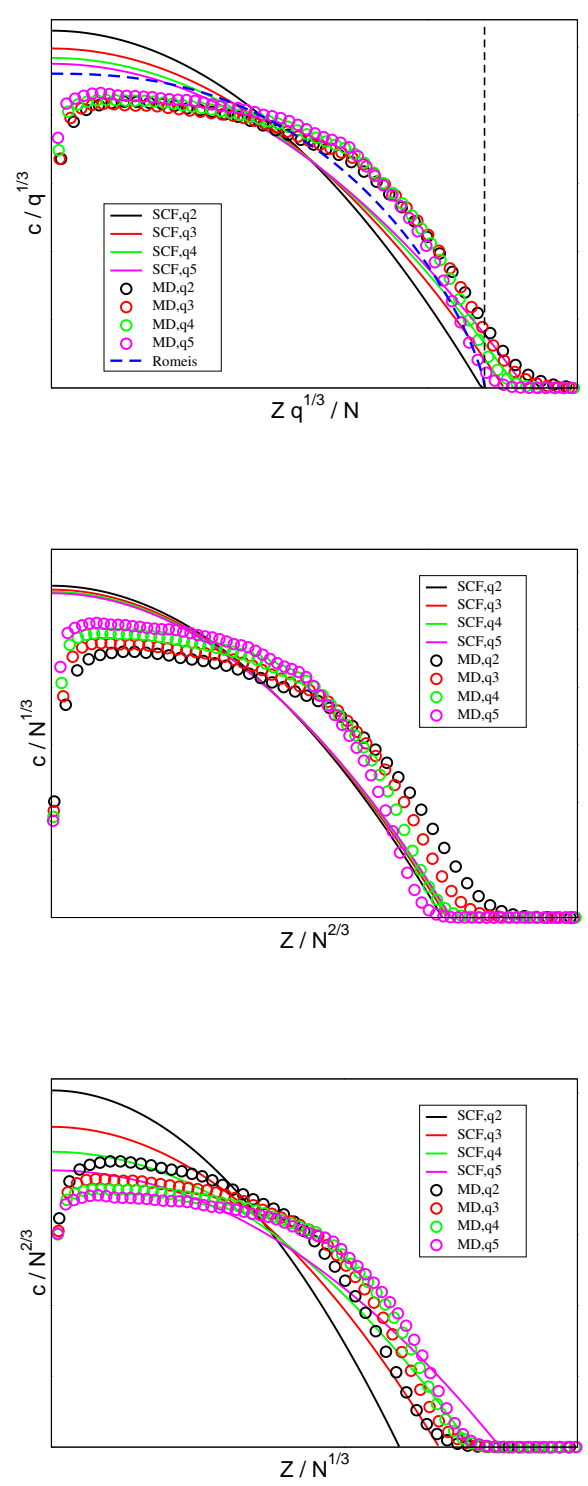

FIG. 2. Density profiles of starlike polymer brushes of different functionality $q$ at low grafting density $\sigma=0.025$. Scaling of the coordinates according to different scenarios: 'all up' stretching model (upper panel), the longest path stretching model (center panel), and the uniform stretching model (lower panel). Spacer length: $N_{S}=100$. The heights of the SCF models (at $q=2$ ) were fitted to the inflection point of the corresponding MD simulation profile (dashed vertical line in upper panel). SCF-predictions are solid curves, the blue dashed curve (upper panel) is the fit of Eq. 24.

$r(q=5)=0.28$. The stretching ratios, averaged over all free branches, remain invariant of the functionality about a constant $r=0.2$. This short analysis excludes the longest path stretching scenario (because the stems are always stretched significantly further out than the branches). The 'all up' scenario is not verified either: If we define an 'all up' conformation as a molecule in which all free ends are located above their branching point, then the fraction of such molecules among the entire population is found to vary between $\lambda(q=$ $2)=0.69$ and $\lambda(q=5)=0.36$, which implies that, with increasing functionality, a diminishing fraction of molecules falls into this scenario.

As a matter of fact, the various stretching scenarios that are assumed in the analytical models are based on overly simplified pictures. The situation is turning even more complex once brushes of higher generations are considered, in which the molecular conformations are increasingly irregular [36]. Yet, simplified models as discussed in the present section are not without their merits: Since they are leading to well defined predictions of the scaling behaviors of brushes, they are helpful in classifying numerical methods which do not a priory predict any scaling behavior. The simple message of Fig. 2, namely that the MD-data approximately scale according to the 'all up' model, while the Gaussian SCF approximately follows the longest path model, may lead to valuable insights into fundamental differences in the physical assumptions onto which these approaches are based.

We further note that the parabolic profiles obtained with the Gaussian SCF method do not generally represent the profiles extracted from the MD simulations well. The latter appear steeper, towards box-like rather than parabolic. In previous works, deviations from the parabolic profile have often been attributed to contributions from higher order repulsive interactions (higher virial coefficients) between monomers [40], or from the finite extensibility of chains $[6,41,42]$. Yet, both contributions should be rather insignificant at the low grafting density considered in Fig. 2, so that additional parameters may lead to the observable deviations from the parabolic shape. A hint comes from the upper panel of Fig. 2, in which we have inserted the profile of Eq. 24. Once more, the brush height $H$ has been fitted to the inflection point (dashed vertical line) of the MD simulation profile at $q=2$. The rather box-like shape of its profile is a result of more realistic assumptions behind the assumed chain's paths, and it does not depend on the density of the brush: There exists no low-density limit that would lead to parabolic brush profiles. The general shortcomings of Gaussian chain based SCF models in situations of strong excluded volume effects have been discussed before by Kreer et al. [43].

Figure 3 displays the brush (center of mass) heights of a large set of 62 systems of different architectures, grafting densities and spacer lengths. These heights are computed by integrating the vertical density profiles $\rho(z)$,

$$
H_{\mathrm{cm}}=\int z \rho(z) d z .
$$

The brushes cover a wide range of (averaged) monomer number densities between $0.05<\langle c\rangle<0.5$, and the heights are scaled according to the 'all up' stretching scenario of Eq. 11. As a function of the spacer length, the brush heights are expected to scale linearly (dashed line, upper panel), and as a function of the grafting density, a scaling of $\sigma^{1 / 3}$ is expected (dashed line, lower panel). Though the overall scaling properties are verified, a good collapse of different architectures onto the same master line is not found. This is no surprise, since 

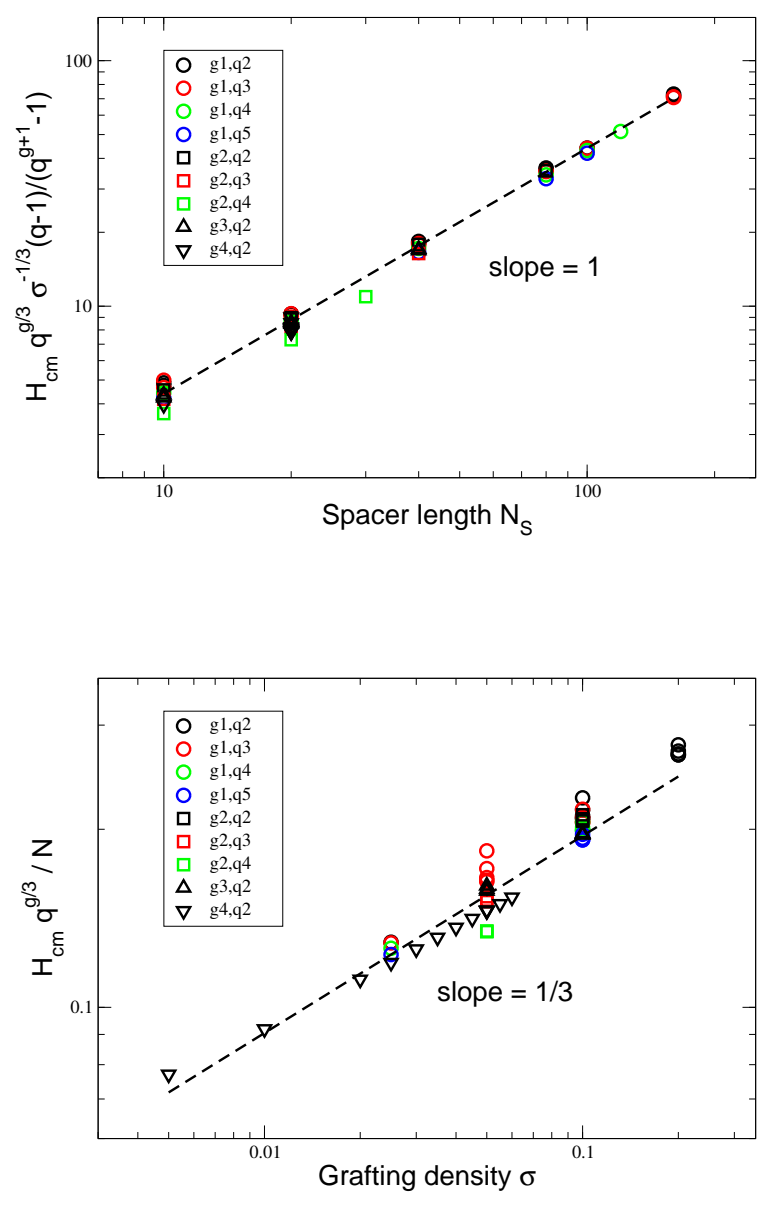

FIG. 3. Brush (center of mass) heights, scaled according to the 'all up' stretching model, as a function of the spacer length $N_{S}$ (upper panel) and the grafting density $\sigma$ (lower panel), for 62 different brushes of various architectures. The dashed lines indicate the respective universal scaling predictions.

many of those brushes shown in Fig. 3 are reaching densities at which the approximations, which led to the analytical Eq. 7, are no longer valid. In particular the harmonic elasticity of the spacers is expected to fail badly for the grafted stem, as soon as the density and/or the number of generations or functionality of the polymer increase. A rather quantitative description of these brushes thus requires models that are more sophisticated, and these will be discussed in the following sections.

\section{DUAL POPULATION BOX MODELS}

Contrary to monodisperse brushes made of linear chains, which contain molecules of qualitatively identical conformations, dendrimer brushes are composed of molecules of qualitatively different conformations. This observation has first been made by Pickett [28], who, with the help of a lattice based numerical SF-SCF method [39], proved that a backfold- ing of branches into lower regions of the brush is practically absent. Consequently, in order to keep the monomer density of the brushes lower parts sufficiently high, a certain fraction of molecules had to retract into a collapsed state. This proposal has been confirmed in subsequent applications of the SF-SCF formalism $[24,29]$ and in MD simulations of brushes in good solvent [35, 44, 45] as well as at the $\Theta$ point [46].

\section{IV.1. Dual phase mean field model}

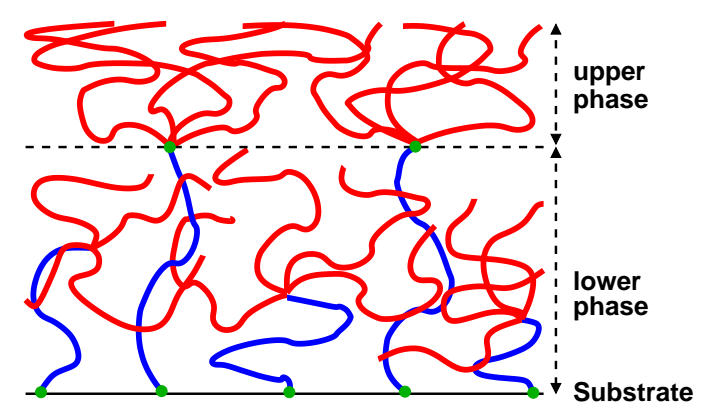

FIG. 4. Schematic illustration of a box model with two phases (here for starlike dendrimers): The upper phase is composed of branches of extended molecules, while the lower phase contains their stems and a suitable fraction of retracted dendrimers.

These observations naturally led to a refinement of the mean field box model (Fig. 4): Instead of assuming a box with a homogeneous population of dendrimers, two different layers are proposed. The upper layer consists of the free branches of extended dendrimers and may be treated like an ordinary linear brush. Depending on the fraction $\lambda$ of extended dendrimers, the upper brush layer contains a certain number of free branches per unit area, hence a 'grafting density', and a standard mean field model for linear brushes is applied to evaluate its thickness and concentration. If a uniform monomer concentration is assumed, then the thickness of the lower layer is immediately known, too. The assumption of a certain stretching mode (in this example taken from the 'all-up' scenario) then leads to a force balance condition at the branching point. The fraction $\lambda$ has to be varied, until both, the uniform monomer concentration and the force balance condition, are satisfied simultaneously.

Since this dual phase box model has to be applied to brushes at moderate and high densities, two notable deviations from the free energy contributions, initially assumed in Sec. II.1, have to be implemented [47]: At high densities, the harmonic approximation for the elasticity of the spacers tends to yield overstretched and hence unphysical spacer lengths. We therefore introduce the non-linear spring force

$$
k(r)=r \frac{3-r^{2}}{1-r^{2}},
$$

a Pade-approximation of the inverse of the Langevin function,

$$
r(k)=\operatorname{coth}(k)-1 / k
$$


which describes ideal chains of fixed contour length in continuum space [48]. Here, $r=h / N_{S}$ is the stretching ratio of the spacer, with a vertical extension of $h$ and a contour length of $N_{s}$. Once the stretching ratio approaches unity, $r \rightarrow 1$, the spring force diverges. Note that at low stretching ratios, the Gaussian approximation $k(r) \approx 3 r$ is restored for both the Langevin equation and its Pade approximation. These functions are plotted in the inset of Fig. 8.

Volumetric interactions in good solvent are taken from the Boublik-Mansoori-Carnahan-Starling-Leland (BMCSL) equation of state for hard-sphere systems $[49,50]$, yielding (in units of $k_{B} T$ ) the osmotic pressure $[51,52]$

$$
\Pi_{\mathrm{BMCSL}}=\frac{1}{V_{\mathrm{m}}}\left[\frac{3 \phi^{2}}{(1-\phi)^{2}}+\frac{\phi^{3}(3-\phi)}{(1-\phi)^{3}}\right] .
$$

Here, $V_{\mathrm{m}}=d^{3} \pi / 6$ is the geometric volume of the monomer of diameter $d$, and $\phi=c V_{\mathrm{m}}$ stands for the volume fraction of the monomers inside the brush. The diameter $d$ may be used as a free parameter to fit the mean field model to molecular simulation data (in what follows we use $d=0.93$ to achieve the best fit to the simulated brush heights). The BMCSL approach leads to beads that are significantly 'harder' than the second order virial term of Flory's mean field approximation [53] and reproduces the MD simulation data well.

With the contributions from Eqs. 28 and 30, the minimization of the resulting mean field model does no longer yield closed expressions for the brush heights, which have to be solved numerically. This is the reason why this class of models has been referred to as being 'semi-analytical' [54]. Elastic contributions to the free energy, originating from the lower phase of retracted dendrimers, are small, but they should be accounted for to yield good results at low grafting densities. As described by Cui [36], it suffices to assume 'all up' conformations for these retracted dendrimers, and a uniform distribution of their ends within the lower phase of the brush. The average elastic contribution of these molecules is then integrated numerically. In that work, the model has as been applied to second generation dendrimer brushes as well.

\section{IV.2. Box model with two-step density profile}

A somewhat modified version of the dual phase boxlike model has recently been presented for starlike brushes by Polotsky et al. [55]. The polymers are described as freely jointed molecules on a lattice. This leads, for a given spacer, to the finite extensible elastic function

$$
r(k)=\frac{\sinh k}{2+\cosh k},
$$

in which, as in Eq. 28, $r$ stands for the stretching ratio, and $k$ for the force pulling at the spacer. The monomer's mixing entropy, excluded volume and additional interactions are accounted for through a Flory-Huggins model. The authors thus arrive at an analytical free energy function, which is minimized numerically to yield the heights, the monomer concentrations of the two layers, and the fraction $\lambda$ of molecules which extend into the upper layer. Note that this model does not rely on the restriction of a uniform monomer density, but it allows for different densities of the layers, so that the resulting brush profile turns into a two-step function.

\section{IV.3. Semi-analytical lattice SCF model}

An interesting approach to starlike brushes at moderate and high grafting densities has been presented by Zhulina et al. [56]: While the ingredients to that model are identical to those presented in the previous section (dual layer, freely jointed polymers on a lattice with non-Gaussian elasticity, Flory-Huggins mixing entropy), the free energy is evaluated self-consistently, yielding not only brush heights and concentrations, but also density profiles. Here, the self-consistent potential for linear chains on a (bcc-)lattice

$$
U_{\mathrm{bcc}}(z)=\Lambda+3 \ln \left(\cos \frac{\pi z}{2 N_{s} a}\right),
$$

is generalized to starlike polymer architectures. Note that this potential is, contrary to Eq. 19, non-parabolic, a consequence of the non-Gaussian elasticity of the spacers. The bond-length $a$ may serve as a free parameter to fit the resulting brush heights to simulation data, and $\Lambda$ is a constant that has to be determined from the boundary conditions. The method results into a set of closed equations, which have to be solved numerically to find the optimum brush height, the density profile and the fraction $\lambda$ of extended molecules [57].

\section{COMPARISON WITH MD SIMULATIONS}

\section{V.1. Brush heights}

Figure 5 shows scaled monomer density profiles of starlike polymer brushes of functionality $q=3$ at different grafting densities $\sigma$. The solid curves are MD simulation data, while the dashed curves are the results of the lattice SCF method described in Sec. IV.3. The scaled MD-profiles are falling rather well onto a master curve. Differences exist near the peripheries of the brushes at which fluctuations play an important role. The SCF curves are consistently ending at a scaled height of $H / \sigma^{1 / 3} \approx 117$, indicating a correct scaling of $H \sim \sigma^{1 / 3}$, but their shapes display systematic variations. Although the brush heights, obtained with the SCF method, are somewhat low when compared to the MD, a minor modification of the bond-length to $a=1.14$ allows to fit them to the (inflection-point) heights of the MD simulations.

Brush heights may be defined in different ways. The center of mass height, as defined in Eq. 27, is a most convenient quantity. In molecular simulations, in which the monomer positions are known, it is computed directly from their vertical coordinates. In boxlike mean field models with uniform monomer density, the trivial relation $H_{\mathrm{cm}}=H / 2$ holds. An alternative way to derive a height from a molecular simulation profile is to locate the inflection point of the profile near 


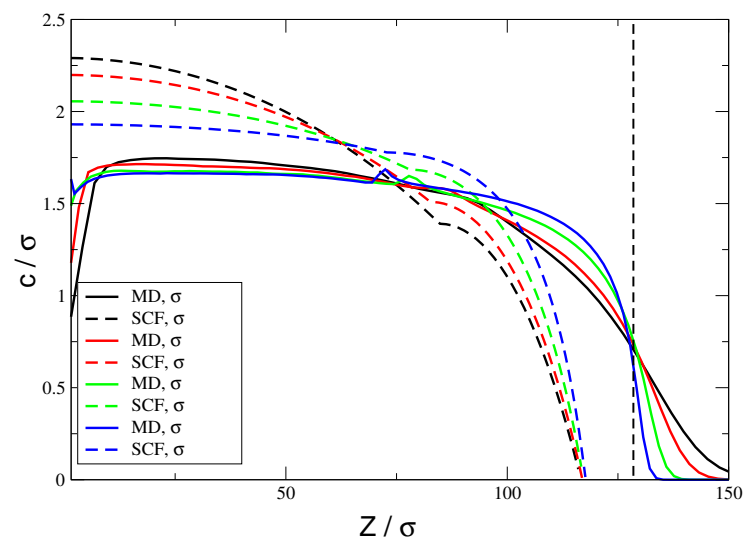

FIG. 5. Density profiles of starlike polymer brushes of functionality $q=3$, scaled with $\sigma^{1 / 3}$ (Eq. 7) and $\sigma^{2 / 3}$ (Eq. 8). The MD simulation data (solid curves) fall onto a master curve, while the lattice SCF profiles of Sec. IV.3 (dashed curves) show systematic changes in their shapes. A choice of $a=1.14$ will later on serve to fit the SCF heights to the inflection points of the MD-profiles (dashed vertical line). Spacer length: $N_{S}=50$.

the brush surface. In Fig. 5 we have indicated the approximate position of the inflection points with the dashed vertical line. The resulting value for $H_{\text {infl }}$ also obeys the $\sigma^{1 / 3}$ scaling law. The disadvantage of this method is that it requires a careful inspection of the profiles and, in order to be accurate, a considerably large sample size. On the contrary, $H_{\mathrm{cm}}$ is computed fully automatically and it is also very robust against statistical fluctuations.

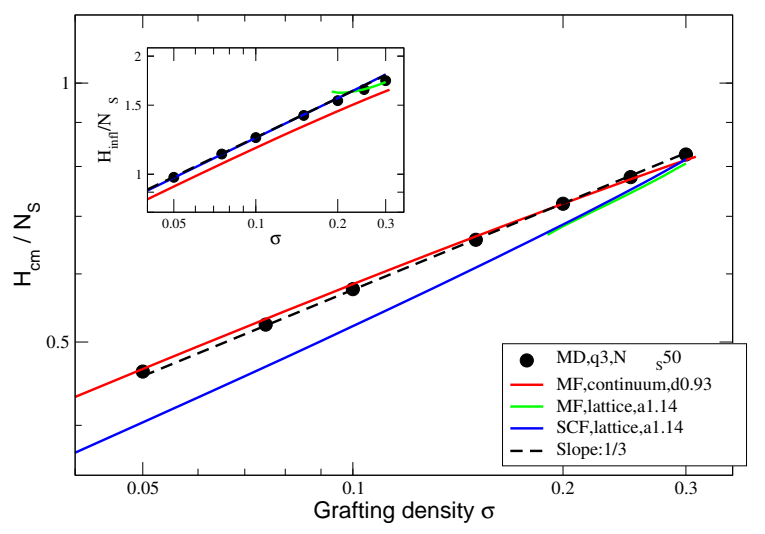

FIG. 6. Center of mass brush heights as a function of grafting density. The continuum space mean field data of Sec. IV.1 (red curve) are fitted to the MD simulation data (dots) and scaling identically. Inset: The lattice SCF data of Sec. IV.3 (blue) are fitted to the brush heights, which have been extracted from the inflection points. The lattice mean field model of Sec. IV.2 (green) yields data only at high grafting densities.
Figure 6 shows that the MD simulation data are consistently scaling with the grafting density, $\sim \sigma^{1 / 3}$, regardless whether the center of mass heights or the inflection points (inset of Fig. 6) are applied. The continuum space mean field model of Sec. IV.1 (red) is fitted to the center of mass data of the MD simulations (because it is a box model with uniform monomer distribution), using an effective monomer diameter of $d=0.93$. With this choice, its total height $H$ is consistently lower than $H_{\text {infl, }}$ as shown in the inset, but the scaling remains accurate in either case. The SCF data display a slope that is too steep, when the center of mass heights are compared. This is a consequence of the fact that the shapes of the SCF profiles in Fig. 5 vary systematically with the grafting density. The locations of the brush surfaces, as shown in the inset, are scaling correctly with the grafting density. The dual layer lattice model of Sec. IV.2 (green) yields the same center of mass heights as the corresponding SCF model, once its bond length is adjusted to the same value $a=1.14$. This method is operating well at high grafting densities, but it is unstable at lower densities, and hence it will not be considered in the following analysis.

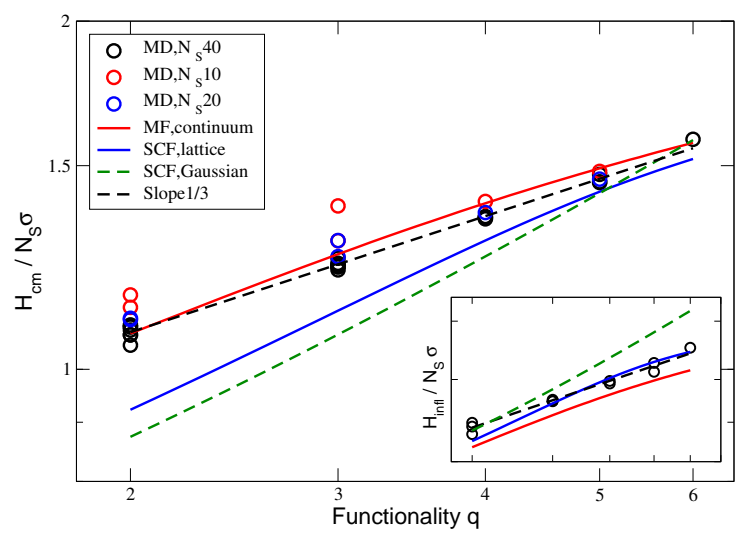

FIG. 7. Brush heights as a function of functionality $q$ for different starlike brushes of various grafting densities and spacer lengths. Scaled center of mass heights (main panel) as well as scaled inflection point positions (inset) of the MD simulations (circles) are compared with the continuum mean field model of Sec. IV.1 (red), the lattice SCF model of Sec. IV.3 (blue), and the analytical Gaussian SCF model of Sec. II.3 (green). The theoretical models were evaluated at a grafting density of $\sigma=0.1$ and with a spacer length of $N_{s}=100$.

Figure 7 represents the main test of the theoretical models of starlike brushes at moderate and high densities: A large number of systems with varying spacer lengths and grafting densities are plotted as a function of their functionality $q$. First focusing on the MD data, we observe that the center of mass heights do not always scale accurately after $H_{\mathrm{cm}} \sim N_{S} \sigma^{1 / 3}$. A breakup into systems of different spacer lengths proves that those brushes with particularly short spacers did not display universality: With short spacers, steric restrictions of the monomers may dominate the conformations which then deviate significantly from their universal shapes. 
The scaling of brushes was violated when the spacer lengths remained below a threshold, which seemed to be of the order of $N_{S} \approx 20$ monomers. Brushes with spacer lengths of at least 40 monomers did not exhibit any signatures for these deviations. For the inset of Fig. 7, only brushes with $N_{S}>40$ were analyzed and the inflection points of their profiles were evaluated manually.

Though non-integer functionalities are not physically meaningful, the mean field or SCF techniques formally allow for an optimization of their equations with non-integer $q$-values. The continuum space mean field model of Sec. IV.1 (red) predicts the center of mass heights of the brushes well. Similarly, the lattice SCF model of Sec. IV.3 (blue) predicts the brush surface heights, evaluated from the inflection points of the MD data profiles, accurately (inset of Figure 7). We note that all theoretical data were obtained using fixed values for the grafting density $\sigma=0.1$ and the spacer length $N_{s}=100$; as long as the universal scaling $H \sim N_{S} \sigma^{1 / 3}$ holds, these parameters are eliminated from the scaled brush heights.

We note that the MD-brush heights seem to yield an approximate power law scaling of $H \sim q^{1 / 3}$ (black dashed line). The simple scaling equation 17 (uniform stretching model) would in fact yield such a scaling, asymptotically at large functionalities $q$, while the 'all up' model of Eq. 11 and the longest path stretching of Eq. 14 would instead yield $H \sim q^{2 / 3}$. This argument should be taken with caution, however, because none of the theoretical models of Sec. II. 2 is expected to remain accurate whenever the Gaussian approximation of the spacer elasticity is breaking down, particularly in the case of large functionalities $q$. The Gaussian analytical SCF model of Sec. II.3 is therefore added to Fig. 7 (dashed green curves) for comparison only: The inset shows that the model coincides with the simulation at the lowest functionality $(q=2)$ : It's excluded volume parameter $v=0.8$ had previously been chosen so that the resulting height was fitting the inflection point of the $q=2$ profile. At higher functionalities, the Gaussian SCF heights rapidly exceed the heights of the simulated brushes and of the finite extensible lattice SCF. We remind that the Gaussian SCF has already been shown to scale approximately according to the longest path scenario (Fig. 2, center panel), and consequently, the slope of the dashed green curve is closer to $2 / 3$ rather than $1 / 3$.

\section{V.2. Conformational features}

The MD data allow for a clear separation between both brush layers sketched in Fig. 4: Since the upper layer exclusively contains monomers of free branches, the phase boundary is defined as the height at which the density distribution of stem-monomers diminishes. At moderate and high densities, the latter distribution displays a steep drop here, and thus the boundary is rather well defined. Dendrimers which have all their end monomers inside the upper layer are defined 'up', those with all ends inside the lower layer are 'down', the remaining dendrimers are in an undefined state [35].

Figure 8 shows the fraction $\lambda$ of starlike molecules that reach into the upper layer of the brush, as a function of graft-

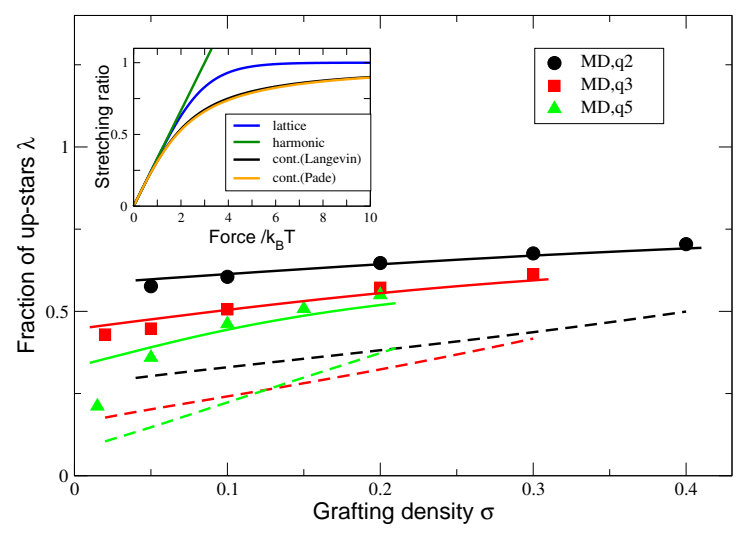

FIG. 8. Fraction $\lambda$ of starlike molecules reaching into the upper layer of the brush, as a function of grafting density and for different functionalities $q$. Symbols: MD simulations. Solid curves: Continuum space mean field theory. Dashed curves: Lattice SCF theory. Inset: The stretching ratio of a spacer as a function of the force, for a harmonic spring (green), a freely-jointed chain on a lattice (Eq. 31, blue), a freely jointed chain in continuum space (Eq. 29, black) and its Pade-approximation (Eq. 28, orange).

ing density and for different functionalities $q$. A comparison between the MD simulation analysis (data points) and the prediction of the continuum space mean field theory of Sec. IV.1 yields a reasonably close agreement, although the data at high functionality and low grafting density begin to deviate significantly. In this regime, it is increasingly difficult to classify polymer conformations from the MD simulation data, since the molecules are turning highly irregular, and a backfolding of single branches into the lower layer no longer remains a rare event. The analytical lattice SCF method (dashed curves) of Sec. IV.3 yields population ratios that differ significantly from the continuum space theory. Yet, the validity of its predictions has been verified through numerical SF-SCF simulations [56]. This implies that the SF-SCF method would generate similarly strong deviations from the MD simulations. A similar observation has been made before during an evaluation of the lattice mean field model [55] (Sec. IV.2). It has been suggested here that the origin of these deviations may be connected to differences in the elastic properties of the spacers: The inset of Fig. 8 shows the stretching ratio $r$ as a function of the force $k$ pulling at a spacer, in three different models: The harmonic spring ( $r=k / 3$, green line), the freely jointed chain on a lattice (Eq. 31, blue curve), and the Pade approximation of the inverse Langevin function for polymers in continuum space (inverse of Eq. 28, orange curve). On the lattice, the spacer initially behaves like a harmonic spring, while rapidly turning stiff at stretching ratios significantly above 0.5 . The continuum space polymer is deviating from the harmonic spring at far lower stretching ratios, yet it does not approach its maximum extension even at forces as high as $10 k_{B} T-$ a fact previously pointed out by Romeis et al. [53]. Hence, in continuum, the polymer is less harmonic under moderate 
forces, but still quite elastic at high forces, while on the lattice the harmonic elasticity is almost instantly crossing over into a fully extended, non-elastic chain.

These facts are likely to have their impact on the population structure of the dendrimers: The bulk of the elastic free energy of the brush is stored inside the stems of the extended molecules. On a lattice, the lower phase can accommodate a larger fraction of molecules than in continuum, in which the increasing thickness of the lower layer causes a rapidly rising free energy penalty from the tension of the stems. Hence, in continuum, a larger fraction of molecules is relocated into the upper layer to minimize the free energy, yielding a higher value of the population fraction $\lambda$.

Despite of the complexity of dendrimer conformations inside brushes, numerical simulations have led to the discovery of certain universal features: SF-SCF simulations have shown that starlike polymers in the lower layer of the brush display a universal vertical distribution profile of their free ends, which does depend on the position of the branching point, but not on the grafting density [29]. Another universality exists for the distribution of the vertical forces pulling at the individual free branches: In MD simulations, the forces acting on every branch of a selected dendrimer (of second generation) were determined, and scaled by the maximum force found in that molecule [36]. The resulting distribution functions differed with the functionality $q$, but they were universal with respect to the grafting density. These two observations may imply that the partition of the elastic tension among free arms, which is eventually related to the stretching scenarios discussed before in Sec. II.2, does not vary with the grafting density. In this sense, the stretching scenarios cannot cross over upon changes of the grafting density.

Similar studies have focused on the degree of backfolding, i.e. the frequence at which individual branches of extended dendrimers are folding back into the lower phase of the brush. Such a scenario is not considered in any of the approximate dual phase brush models discussed in the previous sections. The degree of backfolding was found to be rather low, though increasing with the functionality of the dendrimer [29, 35].

Finally, brushes are not static entities: A dendrimer which occupies the lower phase of the brush may move up into the upper layer to replace another, retracting molecule. Such flipevents have been analyzed in various MD simulation studies $[35,45,46]$. Their kinetics was shown to be dominated by the energy required to stretch up the molecule from the lower into the upper layer. The analysis of the dynamical properties of branched brushes may lead to interesting implications for the design of switchable (environment responsive) layers [2].

\section{SUMMARY AND DISCUSSION}

Brushes made of branched polymers display a degree of complexity that is absent in brushes made of linear chains, such as the spontaneous decomposition into various populations with different degrees of molecular extension. Consequently, a universal theoretical model, which would encompass the full range of architectures at any grafting density, does not yet exist.

In the present work, we have distinguished between methods that describe dendrimer brushes at low densities, and those designed to approximate certain brushes at higher densities. The former yield simple, but reasonably accurate scaling laws (Sec. II.1, II.2), or SCF equations which are written in closed analytical form (Sec. II.3). At higher densities, approximate free energy expressions are available which have to be solved numerically for the brush heights and which remain restricted to a predefined number of generations (Sec. IV.1, IV.2, IV.3). The main technical difference between both classes is rooted in the necessity of nonlinear contributions to the spacer elasticity at moderate or high densities, which complicates the free energy expressions and thus prevents simple analytical solutions. Additionally, the latter models have to account for the dual (or multiple) population structure of the brushes. Their common feature is the assumption of two layers, which contain either extended or retracted dendrimers (Fig. 4).

It appears to be of ample importance to distinguish between the features of lattice-based techniques and continuum space models. The comparison of the population fraction in Fig. 8 yields not only significant, but almost alarming differences in the resulting structures of the brushes. Yet, each of these methods have respectively been validated by numerical procedures, i.e. MD simulations in continuum space, or SF-SCF simulations on the lattice. This implies that even on the level of the numerical procedures, which are lacking most of the simplifying model assumptions, considerably deviating results on certain brush parameters are possible. It has convincingly been argued that differences in the elastic properties of freely-jointed chains on the lattice and in continuum space are likely to be among the main reasons behind the observed deviations [36, 55].

The scope of this work had to be limited to a small subset of systems which were studied in recent years. The focus was on brushes in good solvent. At the $\Theta$-point, Eq. 7 is, in leading order of the virial expansion, replaced by

$$
H=\left(\frac{\mathrm{w} N^{3}}{K}\right)^{1 / 4} \sigma^{1 / 2},
$$

but the scaling of $H \sim \sigma^{1 / 2}$ has not been observed in MD simulations of brushes in most common regimes of grafting densities, neither in the case of linear nor dendrimer brushes $[46,58]$. Contrary to brushes in good solvent, the leading order term of the virial expansion, Eq. 2, appears to be insufficient to account for the volumetric interactions inside brushes, since they are naturally less swollen and thus of higher monomer concentration. Simulations at very low grafting densities did approach the above scaling law, and mean field models with higher order virials were employed to verify the characteristic crossover behavior of brushes at the $\Theta$ point [58].

In additional related works, the scaling laws of dendrimer brushes on curved surfaces have been studied and compared to numerical SF-SCF simulations [10, 25]. Dendritically branched polyelectrolytes have been analyzed using meanfield and SCF theory in a recent study [59], and the properties 
of mixed brushes, made in parts of dendrimers and in parts of linear polymers, has been analyzed, too [1]. The analytical SCF model of dendrimer brushes is meanwhile generalized to polydisperse molecules [30], and brushes made of comblike polymers have been studied in scaling theory [22] and in numerical SCF simulations [7]. It should be added that (linear) brushes at high densities have also been successfully treated with density functional theory $[60,61]$.

While the continuum space mean field model of Sec. IV.1, and its extension to second generation dendrimer brushes [36], predict brush heights accurately, they do not deliver density profiles. Future improvements may thus focus on the SCF modeling of branched brushes. The lattice-SCF model of Sec. IV.3 is capable of predicting the heights of starlike polymer brushes, obtained in MD simulations, accurately, as long as the surfaces of the brushes are compared. However, it yields center of mass heights that are not only deviating quantitatively from MD simulations, but also violating the scaling predictions for different grafting densities (Fig. 6, blue curve). This is a result of systematic variations of the shapes of its density profiles (Fig. 5). The analytical SCF theory of Sec. II.3 leads to profiles that are parabolic, while the simulated profiles are, even at low grafting densities, rather boxlike (Fig. 2). Additionally, it scales closely according to the longest path stretching scenario (center panel of Fig. 2) - a behavior not observed in MD simulations.

The semi-analytical SCF model of Sec. II.4 is based on the assumption of self-avoiding random walks and yields more accurate profiles, but it is currently restricted to linear chain brushes and to low grafting densities. Shim and Cates have presented an SCF formalism for brushes of linear chains, which includes the finite extensibility and yields rather box- like shapes [62]. Unfortunately, it contains an empirical parameter which has been observed to depend on the grafting density [63]. Rather promising appears the continuum space SCF model (of linear brushes) designed by Biesheuvel et al. [42], which is making use of the non-parabolic SCF potential

$$
U(z)=2 \frac{z^{2}}{a} \frac{2-\frac{4}{5} z^{2}}{1-z^{2}}
$$

with the Kuhn length $a$. The monomer interactions are of the modified Carnahan-Starling type as presented in Eq. 30. Its density profiles are in close agreement with MD simulations, including charged brushes [42, 64, 65]. A generalization of this semi-analytical model to branched brushes might lead to a fairly simple, yet versatile SCF formalism in continuum space, which would avoid the restrictions imposed by the Gaussian approximation of the spacer elasticity.

As shown by Romeis et al, it is also possible to upgrade lattice SCF methods to 'quasi-continuum' models through a refinement of the applied lattice constant [53]. The elastic properties of the polymers are then crossing over into their continuum limits, too, so that modified SF-SCF procedures appear feasible which then would avoid the restrictions imposed by the lattice representation of the polymers. As an additional benefit, these hybrid lattice/continuum models could serve as valuable tools to study the impact of lattice effects on the structures of branched polymer brushes.

Acknowledgments: This work was partly supported by the National Science Foundation of China under Grant No. 11274258 , the 111 project (B16029), and the DFG priority program SPP 1369.
[1] Klushin, L. I.; Skvortsov, A. M.; Polotsky, A. A.; Qi, S.; Schmid, F., Phys. Rev. Lett. 2014, 113, 068303.

[2] Liu, F.; Urban, M. W., Progress in Polymer Science 2010, 35, 3.

[3] Uhlmann, P.; Merlitz, H.; Sommer, J.-U.; Stamm, M., Macromolecular Rapid Communication 2009, 30, 732.

[4] Merlitz, H.; He, G.-L.; Wu, C.-X.; Sommer, J.-U., Phys. Rev. Lett. 2009, 102, 115702.

[5] Merlitz, H.; He, G.-L.; Sommer, J.-U.; Wu, C.-X., Macromolecules 2009, 42, 445.

[6] Merlitz, H.; He, G.-L.; Wu, C.-X.; Sommer, J.-U., Macromolecules 2008, 41, 5070.

[7] de Vos, W. M.; Leermakers, F. A. M.; Lindhoud, S.; Prescott, S. W., Macromolecules 2011, 44, 2334.

[8] Irvine, D. J.; Mayes, A. M.; Griffith-Cima, L., Macromolecules 1996, 29(18), 6037.

[9] Gillich, T.; Benetti, E. M.; Rakhmatullina, E.; Konradi, R.; Li, W.; Zhang, A.; Schlüter, A. D.; Textor, M., Journal of the American Chemical Society 2011, 133(28), 10940.

[10] Rud, O. V.; Polotsky, A. A.; Gillich, T.; Borisov, O. V.; Leermakers, F. A.; Textor, M.; Biershtein, T. M., Macromolecules 2013, 46, 4651.

[11] Button, B.; Cai, L.-H.; Ehre, C.; Kesimer, M.; Hill, D. B.; Sheehan, J. K.; Boucher, R. C.; Rubinstein, M., Science 2012, 337, 937.
[12] Bon, I.; Lembo, D.; Rusnati, M.; Cl, A.; Morini, S.; Miserocchi, A.; Bugatti, A.; Grigolon, S.; Musumeci, G.; Landolfo, S.; Re, M. C.; Gibellini, D., PLoS ONE 2013, 8(10), e76482.

[13] Vincent, L.; Varet, J.; Pille, J.-Y.; Bompais, H.; Opolon, P.; Maksimenko, A.; Malvy, C.; Mirashi, M.; Lu, H.; Vannier, J.P.; Soria, C.; Li, H., International Journal of Cancer 2003, 105(3), 419.

[14] Devaux, C.; Cousin, F.; Beyou, E.; Chapel, J.-P., Macromolecules 2005, 38, 4296.

[15] Zhang, A.; Zhang, B.; Wächtersbach, E.; Schmidt, M.; Schlüter, A. D., Chemistry A European Journal 2003, 9(24), 6083.

[16] Zhang, B.; Wepf, R.; Fischer, K.; Schmidt, M.; Besse, S.; Lindner, P.; King, B. T.; Sigel, R.; Schurtenberger, P.; Talmon, Y.; Ding, Y.; Kröger, M.; Halperin, A.; Schlüter, A. D., Angewandte Chemie International Edition 2011, 50(3), 737.

[17] Andreopoulou, A.; Carbonnier, B.; Kallitsis, J.; Pakula, T., Macromolecules 2004, 37, 3576.

[18] Pasquino, R.; Zhang, B.; Sigel, R.; Yu, H.; Ottiger, M.; Bertran, O.; Aleman, C.; Schlüter, A. D.; Vlassopoulos, D., Macromolecules 2012, 45(21), 8813.

[19] Alexander, S., J. Phys. (France) 1977, 38, 977.

[20] deGennes, P., Macromolecules 1980, 13, 1069.

[21] Zhulina, E.; Vilgis, T., Macromolecules 1995, 28, 1008.

[22] Kröger, M.; Peleg, O.; Halperin, A., Macromolecules 2010, 43, 
6213.

[23] Rubinstein, M.; Colby, R. H., Polymer Physics, Oxford University Press, 2004, Page 100.

[24] Polotsky, A. A.; Gillich, T.; Borisov, O. V.; Leermakers, F. A. M.; Textor, M.; Birshtein, T. M., Macromolecules 2010, $43,9555$.

[25] Borisov, O.; Polotsky, A.; Rud, O.; Zhulina, E.; Leermakers, F.; Birshtein, T., Soft Matter 2014, 10, 2093.

[26] Boris, D., Macromolecules 1996, 29, 7251.

[27] Klos, J.; Sommer, J.-U., Macromolecules 2009, 42, 4878.

[28] Pickett, G. T., Macromolecules 2001, 34(25), 8784.

[29] Polotsky, A. A.; Leermakers, F. A. M.; Zhulina, E. B.; Birshtein, T. M., Macromolecules 2012, 45(17), 7260.

[30] Zhulina, E. B.; Leermakers, F. A.; Borisov, O. V., Langmuir 2015, 31, 6514.

[31] Zhulina, E.; Leermakers, F.; Borisov, O., Scientific and Technical Journal of Information Technologies, Mechanics and Optics 2015, 15, 493.

[32] Zhulina, E. B.; Leermakers, F. A. M.; Borisov, O. V., Macromolecules 2015, 48, 8025.

[33] Romeis, D.; Lang, M., Journal of Chemical Physics 2014, 141, 104902.

[34] Kremer, K.; Grest, G., J. Chem. Phys. 1990, 92, 5057.

[35] Merlitz, H.; Wu, C.-X.; Sommer, J.-U., Macromolecules 2011, 44, 7043.

[36] Cui, W.; Su, C.-F.; Merlitz, H.; Wu, C.-X.; Sommer, J.-U., Macromolecules 2014, 47, 3645.

[37] Weeks, J.; Chandler, D.; Anderson, H., Journal of Chemical Physics 1971, 54, 5237.

[38] Plimpton, S., J. Comp. Phys. 1995, 117, 1, Http://lammps.sandia.gov/.

[39] Fleer, G.; Cohen-Stuart, M.; Scheutjens, J.; Cosgrove, T.; Vincent, B., In Polymers at Interfaces. Chapmann and Hall, London, 1993 .

[40] Zhulina, E.; Borisov, O.; Pryamitsyn, V.; Birshtein, T., Macromolecules 1991, 24, 140.

[41] Amoskov, V. M.; Pryamitsyn, V. A., J. Chem. Soc. Faraday Trans. 1994, 90, 889.

[42] Biesheuvel, P. M.; de Vos, W. M.; Amoskov, V. M., Macromolecules 2008, 41(16), 6254.

[43] Kreer, T.; Metzger, S.; Müller, M.; Binder, K.; Baschnagel, J.,
J. Chem. Phys. 2004, 120, 4012.

[44] Gergidis, L. N.; Kalogirou, A.; Vlahos, C., Langmuir 2012, 17176.

[45] Cui, W.; Su, C.-F.; Wu, C.-X.; Merlitz, H.; Sommer, J.-U., J. Chem. Phys. 2013, 139, 134910.

[46] Su, C.-F.; Cui, W.; Merlitz, H.; Wu, C.-X.; Sommer, J.-U., Polymer 2014, 55, 3254.

[47] Merlitz, H.; Cui, W.; Wu, C.-X.; Sommer, J.-U., Macromolecules 2013, 46, 1248.

[48] Cohen, A., Rheologica Acta 1991, 30, 270.

[49] Mansoori, G. A.; Carnahan, N. F.; Starling, K. E.; Leland, T. W., Journal of Chemical Physics 1971, 54, 1523.

[50] Boublik, T., Journal of Chemical Physics 1970, 53, 471.

[51] de Vos, W. M.; Biesheuvel, P. M.; de Keizer, A.; Kleijn, J. M.; Cohen-Stuart, M. A., Langmuir 2008, 24, 6575.

[52] de Vos, W. M.; Biesheuvel, P. M.; de Keizer, A.; Kleijn, J. M.; Cohen-Stuart, M. A., Langmuir 2009, 25, 9252.

[53] Romeis, D.; Merlitz, H.; Sommer, J.-U., Journal of Chemical Physics 2012, 136, 044903.

[54] Biesheuvel, P. M., Journal of Colloid and Interface Science 2004, 275, 97.

[55] Polotsky, A. A.; Misorin, A. K.; Zhulina, E. B.; Birshtein, T. M., Macromol. Symp 2015, 348, 33.

[56] Zhulina, E.; Amoskov, V.; Polotsky, A.; Birshtein, T., Polymer 2014, 55, 5160.

[57] We note that Eq. (17) in that work has a minor but influential error: The right hand side should read $\arctan (\ldots)$ instead of $a \tan (\ldots)$.

[58] Merlitz, H.; Wu, C.-X.; Sommer, J.-U., Macromolecules 2012, 45, 8494.

[59] Borisov, O.; Zhulina, E., Macromolecules 2015, 48, 1499.

[60] Egorov, S., Phys. Rev. E 2005, 72, 010401(R).

[61] Egorov, S.; Romeis, D.; Sommer, J.-U., J. Chem. Phys. 2012, 137, 064907.

[62] Shim, D.; Cates, M., J. Phys. France 1989, 50, 3535.

[63] He, G.; Merlitz, H.; Sommer, J.; Wu, C., Macromolecules 2007, 40, 6721 .

[64] He, S.-Z.; Merlitz, H.; Chen, L.; Wu, C.-X.; Sommer, J.-U., Macromolecules 2010, 43, 7845.

[65] Chen, L.; Merlitz, H.; He, S.-Z.; Wu, C.-X.; Sommer, J.-U., Macromolecules 2011, 44, 3109. 
* Improved theoretical models of dendrimer brushes are now available

* We test their scaling properties and compare them with MD simulations

* Some brush properties differ significantly in these models

* We attribute these differences to the elastic properties of the molecules

* We discuss methods that might lead to further improved representations 


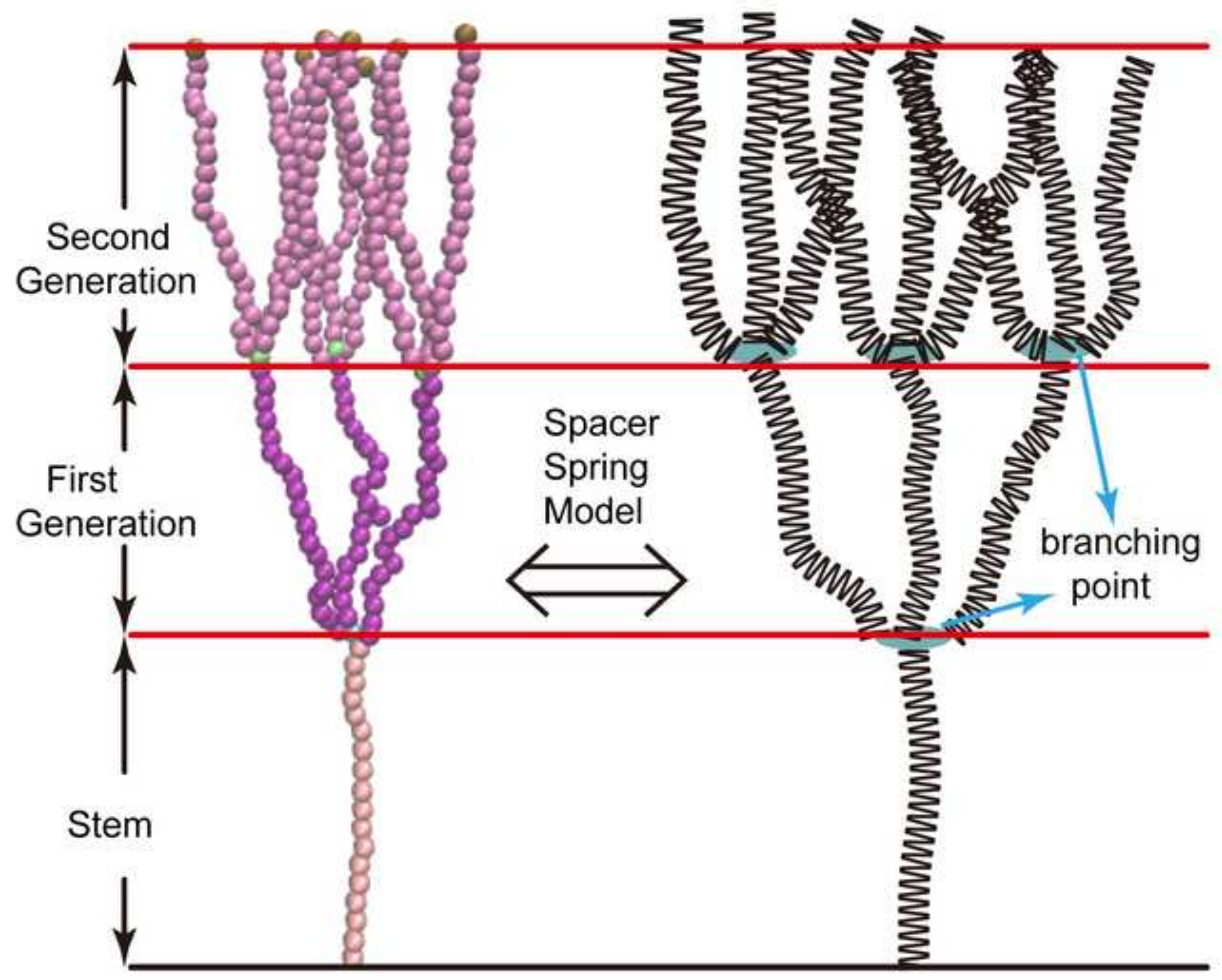

